\title{
Proposing an Interoperable Framework among Cloud Providers
}

\author{
Yasaman Mirfakhrai \\ Computer Engineering \\ Department, Faculty of \\ Engineering, \\ Science \& Research Branch, \\ Islamic Azad University, \\ Tehran, Iran
}

\author{
Mehran Mohsenzadeh \\ Computer Engineering \\ Department, Faculty of \\ Engineering, \\ Science \& Research Branch, \\ Islamic Azad University, \\ Tehran, Iran
}

\author{
Seyed Mohsen Hashemi \\ Computer Engineering \\ Department, Faculty of \\ Engineering, \\ Science \& Research Branch, \\ Islamic Azad University, \\ Tehran, Iran
}

\begin{abstract}
The popularity of cloud computing has increased in recent years and huge Information Technology companies like Google, Amazon, Microsoft and IBM each have launched their own cloud infrastructures which have resulted in individual and island-like infrastructures. The clients of cloud services are growing and various businesses, organizations and individuals use cloud services for their different purposes. The tendency to use multiple providers simultaneously by the same client, migration from one cloud provider to another one and creating composite services using the provided services by various cloud providers, increases the need for interoperability among them. Using the concepts of interoperability in organizations, this research aims at proposing a framework which without any impact on the infrastructures and cloud providers' platforms facilitates the interaction among them. This research discusses high-level issues of interoperability among cloud providers and helps architects to have an organized viewpoint about them.
\end{abstract}

\section{General Terms}

Cloud Computing

\section{Keywords}

Cloud Computing, Interoperability, Interoperability framework, Service Composition, Service Interaction, Lock-in.

\section{INTRODUCTION}

In the cloud computing, interoperability is considered as a key requirement. Cloud computing clients are eager to move freely among the clouds. Interoperability allows the clients to switch among providers based on their needs and enables them to transfer their applications and workloads based on the change in their business requirements. No real interoperability among clouds has been done yet. The standards are being created and this process will take so many years. Joe Skorupa, the deputy of Gartner states, [1]" if, even one open cloud standard is established, all of the providers will continue to develop their own applications in order to create a difference between their own product and those of their rivals. He also states that the cloud providers are not eager to make their products compatible, because in that case the price would be the only factor for competition. In the real world, asking the cloud provider to be consistent with specific models and standards seems unreal or at least hard to achieve. The competition and the reality that differences leads to positive competitions, organizational reasons and technical obstacles, hinder the cloud providers from fundamentally reconstructing the infrastructures. To overcome the interoperability issues among the cloud providers, a flexible approach is required. Focusing on the present concepts in different areas of interoperability, especially in organizations, and also using the available models, approaches and frameworks and considering the concepts of services in cloud computing, this research aims at proposing a framework which without making any change in infrastructures of the cloud providers, fixes the issues of interoperability and interactions among them.

Interoperability among the cloud providers can be discussed from three different major perspectives:

- Technical interoperability: which is related to the Interoperability of the systems and tries to support the interoperability of the systems using the standards, translators, bridges and mapping among protocols.

- Syntactic interoperability: format of the exchanged data and services among systems should be understandable for them. This type of interoperability is the prerequisite for more interoperability.

- Semantic Interoperability: capability of the computer systems to interpret the exchanged information in the receiver system in such a way that is compatible with the concept that is meant by the transmitter.

\section{RELATED WORKS}

Interoperability is a vital aspect of software and systems engineering. This word is used a lot in the literature but, depending on the area of usage, it will be defined differently [2].

The accepted definition which is used in this research is defined by the IEEE:

"It is referred to the ability of multiple systems or components in exchanging information and using the information which are exchanged"[3].

Given the fact that the number of cloud providers are increasing every day, the need for the presence of interoperability among them is strongly felt. The main cloud providers like Microsoft and Amazon, do not conform to the agenda of CCIF and follow their own standards. This issue makes the standardization and conformity absolutely difficult and ends in multiple standards [4].

Two approaches for interoperability at high level are exist:

Approach 1: Cloud broker: cloud broker is trying to integrate different cloud interfaces and clients access to providers only via integrated cloud interface. This approach only related to 
APIs, therefore only data and processes define in this layer are accessible.

Approach 2: Enterprise Cloud Orchestration platform: is related to create orchestration of services. In this approach different cloud providers can register their services and cooperate in orchestration. This orchestration layer can select and bind services based on specific criteria and algorithms dynamically, therefore the best services for doing specific job will be select.[5]

In order to create interoperability, many different institutions are working to create standards. One of these institutions is DMTF, which by standardizing the interactions among cloud environments with creating cloud resource management protocols, packaging formats and security procedures, facilitates the interoperability. [6]

Based on the definition proposed by IEEE, in order to create interoperability among cloud providers, at least three types of conversions are needed for the exchanged information to be understandable technically, syntactically and semantically.

The first type is related to the technical interoperability which is itself consists of two parts, the first part covers the subjects which are related to the connections of the computer systems and contains key aspects like open interfaces, services connections, data and middleware integration, data exchange and display, accessibility and service security. This definition includes the standards and protocols from all seven layers of the OSI reference model. Since, the exchangeable components among the cloud providers are the services, from a practical viewpoint it would be clear that first the physical connection among the cloud providers should be established and the physical platforms should be ready for the constitutive Bits and Bytes of the services to be transferred for migration or interaction. This process is currently possible with the help of some internet connection standards which are available like HTTP, TCP/IP, UDP/IP and etc. and also the internet communication platforms which provide the possibility of transforming symbols and data. Sending the Bits is the only thing that is guaranteed in this section, and nothing will be stated about the semantic of the Bits and what they display. The second part of the technical interoperability is related to the conversion of different protocols and standards which are used by the providers. Practically, using the same protocol by cloud providers has a lot of restrictions and is not performable. Therefore, in order to convert the protocols to each other, there should be facilities to convert communication protocols in a framework. In technical conversion interface, it is possible to use the architectures like Message Hub or Federated Message Hub in a more professional level.

When the possibility of transferring the services is provided, then it comes to define the format of the exchanged information in a way that the service is syntactically understandable for another provider. To achieve this objective, the format of the services should be converted in order to overcome the formats mismatch of data and services. Using XML, interface description languages, CORBA and SOAP and establishing a syntactic translator and adaptor mechanisms, syntactic interoperability will be achieved and services will be syntactically understandable.

Now, it is the time for the exchanged service to be semantically understandable. In fact, at this level data is converted to information and without interpreters, cloud providers are not able to understand each other. Ontology mapping is a necessary prerequisite for creating interoperability among services which are using different ontologies. Defining the ontologies and creating a mapping and a glossary, this level of interoperability will also be achieved. At this level, organizational ontologies like REA, e3-Value and e-BMO can be used and by using ontologies like REA the mapping among them can be done. Thus, semantics at different level will be mapped to each other and will be understandable for the target cloud provider or organization.

Having three aforementioned levels, the minimum requirements for establishing the interoperability will be provided. As is stated in [2,7] (Levels of Interoperability Model) higher levels of interoperability are more related to the organizational and human issues and according to the definition of interoperability, services/information should be exchanged and must be understandable. Eventually, by creating technical interoperability, services will be transferred and with having syntactic and sematic interoperability, they will be understandable for cloud providers and organizations.

\section{INTEROPERABILITY FRAMEWORK AMONG PROVIDERS}

The following conceptual framework is called interoperability framework among providers. The aim of this framework is to create interoperability at the time of migration, interaction and system composition among cloud providers. The concepts of interoperability in organizations are used in this framework.

\subsection{New definitions}

Before you begin to format your paper, first write and save new definitions are required in the proposed framework:

Definition 1: cloud organization: is referred to an organization which has one of the following criteria:

a) Cloud organization is referred to a number of cloud providers which there services are used for orchestration and creating composite services with different performance or executing a process model or a workflow requested by the client. This cloud organization is established temporarily, in order to achieve an objective. The definition that is proposed

Here is close to the definition of virtual organization. The difference between cloud organization and virtual organization is that in virtual organization there is no boundary among the components which are providing services and there is equal participation, while in cloud organization the boundary among the service providers are clear-cut and participation is not necessarily equal and depends on the defined policy by the central unit.

b) Cloud organization is referred to the cloud providers which use the same standards, laws and regulations. This cloud organization can be formed in different levels like technical, syntactic and semantic level.

Definition 2: cloud world: is referred to all of the present cloud providers which according to the needs of their users or 


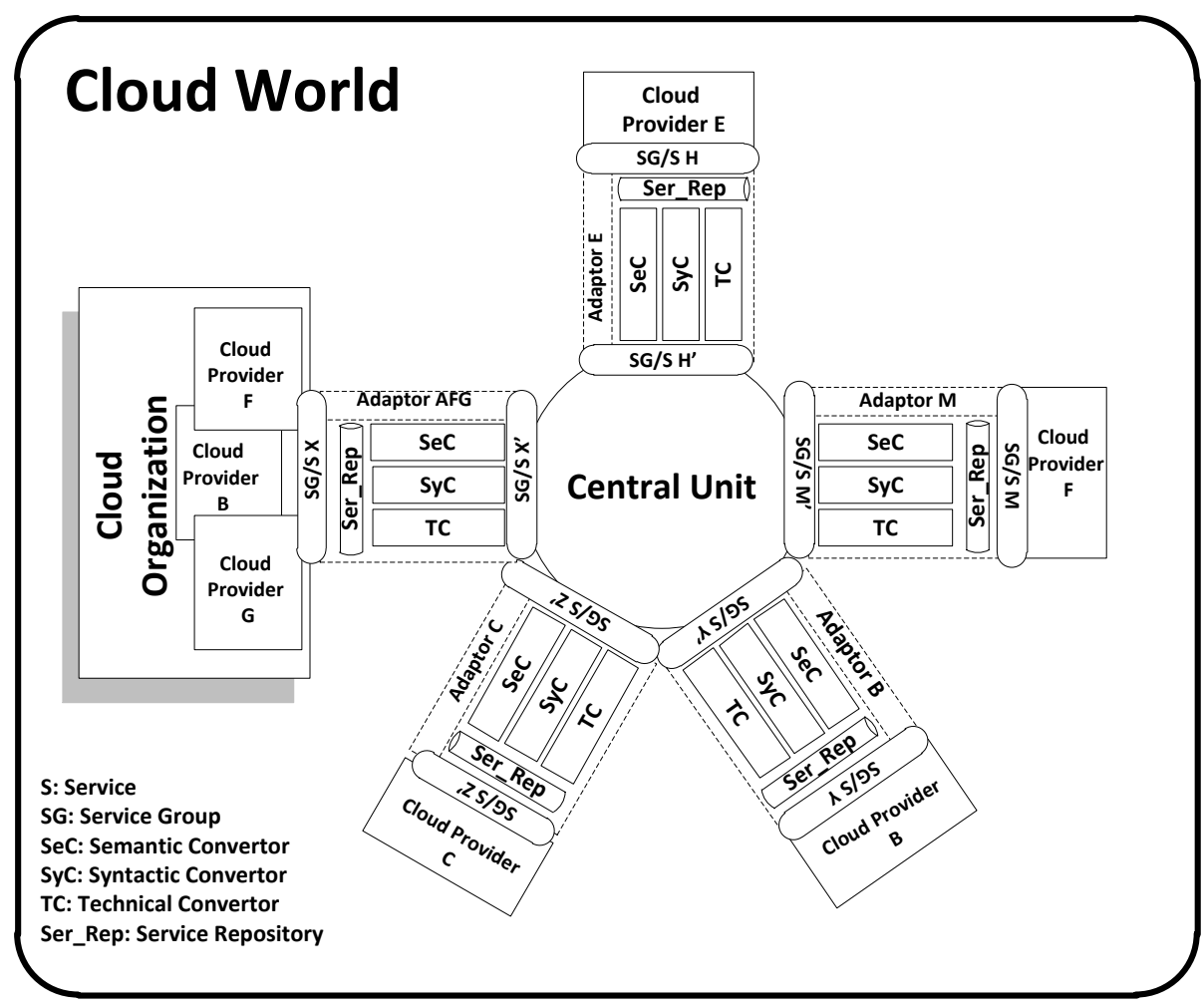

Fig. 1:Overview of Interoperability Framework among Cloud Providers

their own needs interact with each other through the central framework. Each of the cloud providers follows their own standards, laws and regulations.

\subsection{Outline}

The main objectives for creating interoperability among cloud providers in the proposed framework are as follows:

\section{a) Service migration \\ b) Service interaction \\ c) Service composition}

Fig. 1 outlines the proposed framework for interoperability among the cloud providers.

Cloud interoperability framework is made up of three major components namely, cloud providers/organizations, adaptor and central unit which are explained below.

- Cloud providers/organizations: which are responsible for providing services; cloud organizations provide composite services.

- Adaptors: in the proposed framework, in order not to impose any change the current situation of the providers and for the proposed framework to be built based on their current situation, an adaptor is predicted per each or a number of cloud providers/ organizations, that establishes the communication with the central unit, which itself consists of two parts, the first one is the service repository and the other one is the conversion section that is proposed below:

Service Repository: is the section in which description of services which may participate in orchestration and composition of the services from provider's side are placed there.
Conversion section: this section consists of three sub sections in which the conversions are done in three technical, syntactic and semantic levels on the services in order to make them understandable for the central unit. The technical conversion section deals with the issues related to the connectivity of the provider to the central unit and converting the protocols between these two. Using the defined rules by the central unit, the syntactic conversion section does the required mappings in order to prepare the service to be used by the central unit. Using translators and ontologies, semantic conversion section interprets the meanings for the central unit.

- Central unit: in this section, standards, policies, rules and languages which the adaptors of each cloud provider/organization must comply, are defined. Furthermore, some controls for the compatibility of the source provider and target provider's standards are done in this section and some parts of the required conversions and mappings will be done in this section, if necessary.

Defined standards by the central unit are varied and are continuously updating with new rules, regulations, languages and mappings, which eventually makes the interactions less complicated.

\subsection{Selected model and approach in interoperability}

Interoperability models are discussed in [8], and as is stated there, there are three models for establishing the connection. Considering the fact that in the defined cloud world, there are many providers which have their own standards and rules, the central interface cannot have all of the required knowledge to establish the link among the providers, hence, the central unit 


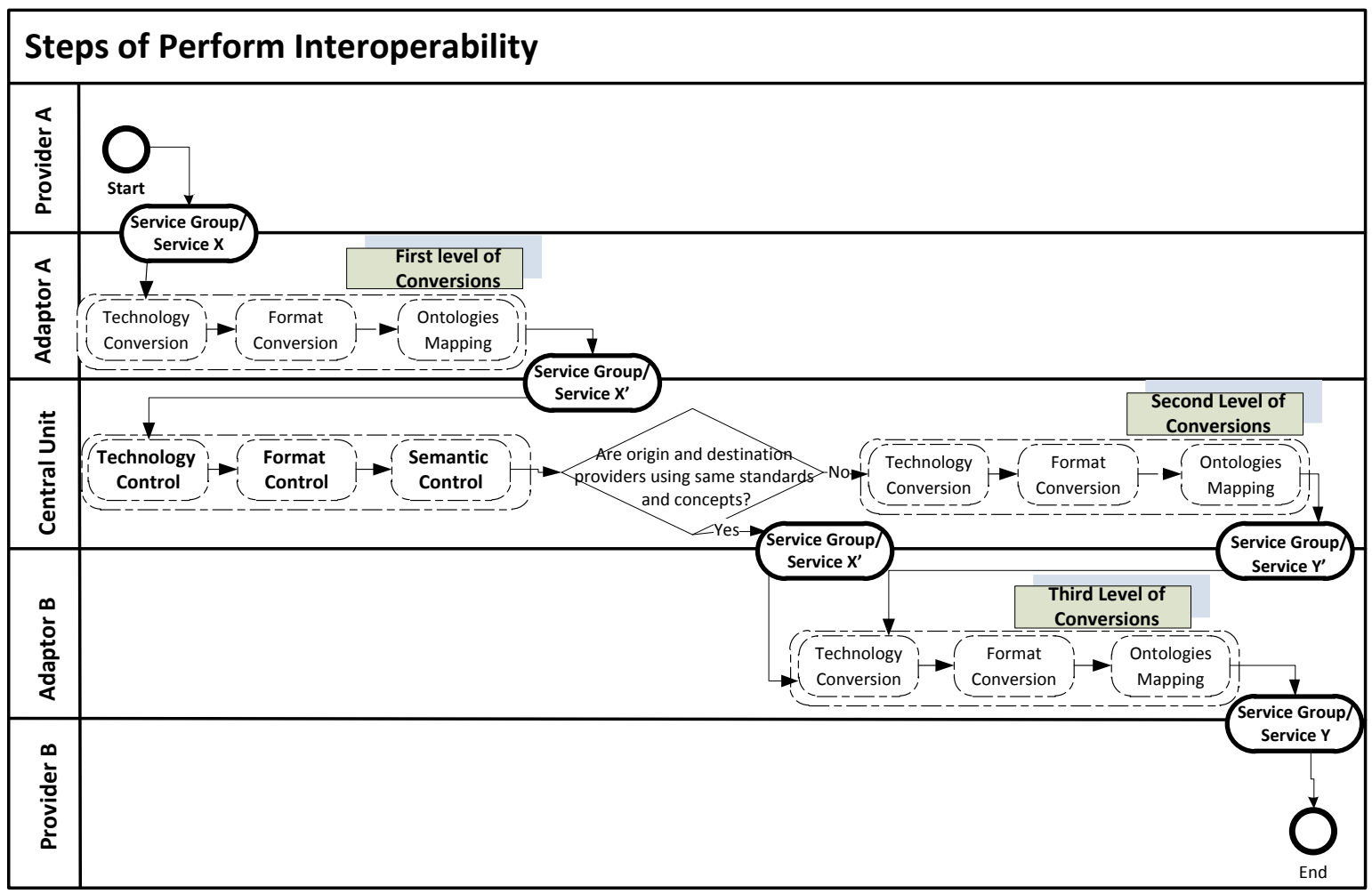

Fig. 2: Interoperability Steps

Model must be used. Since establishing an interaction is not possible without trust, like the central unit model in this model also the trust will be provided among providers and the central unit will play the role of a trusted third party. In [3, 9] interoperability approaches are discussed comprehensively. In order to create interoperability and interaction among the cloud providers and also orchestration for executing a workflow using the provided services by the cloud providers, two types of mutual relationship will be present in the proposed framework:

\section{- Cloud Provider/ organization-adaptor}

\section{- $\quad$ Adaptor-central unit}

In cloud Provider/organization-adaptor relationship, because the adaptor is aware of the details of the standards used by cloud organization/ provider and is able to have a pre-defined meta-model of the mapping between cloud provider/ organization standards and defined standards by the central unit, the unified approach will be used. In the relationship between the adaptor and the central unit, due to the fact that adaptor follows the defined policies, languages and rules by the central unit, the interaction between them is done through an integrated approach. As already discussed, the central unit establishes the possibility for migration between cloud providers, interaction among services and also service composition through orchestration. All of the mentioned items will be covered by two mutual links between provider-adaptor and adaptorCentral Unit.

\subsection{How to perform interoperability}

Considering the selective models and approaches, this section will discuss the way interactions are done in the cloud world. Service migration or interaction through central interface between two providers is done as follows:

1. The provider A, through the Unified approach, sends the service which is going to be migrated or interacted with another provider to the adaptor. The adaptor that is an interface between central unit and provider A, is technically the input and output zone for the provider to establish the interoperability. This interface performs the technical, syntactic and semantic conversion of the service for the central unit and makes the service understandable. Interoperability between the adaptor and central unit is done through an integrated approach.

2. When the service is delivered to the central unit with an understandable format, then the target cloud provider must be searched and found and negotiations should be done between target cloud provider and central unit. Based on the agreement, some technical, syntactic and semantic controls must be done on the service and in case the source and target adaptors had used the same defined standards, rules and regulations by the central unit, the packages will be delivered to the adaptor of the target cloud provider, otherwise, some conversions and mappings will be done in the central unit in order to make the service understandable for the adaptor of the target cloud provider. 


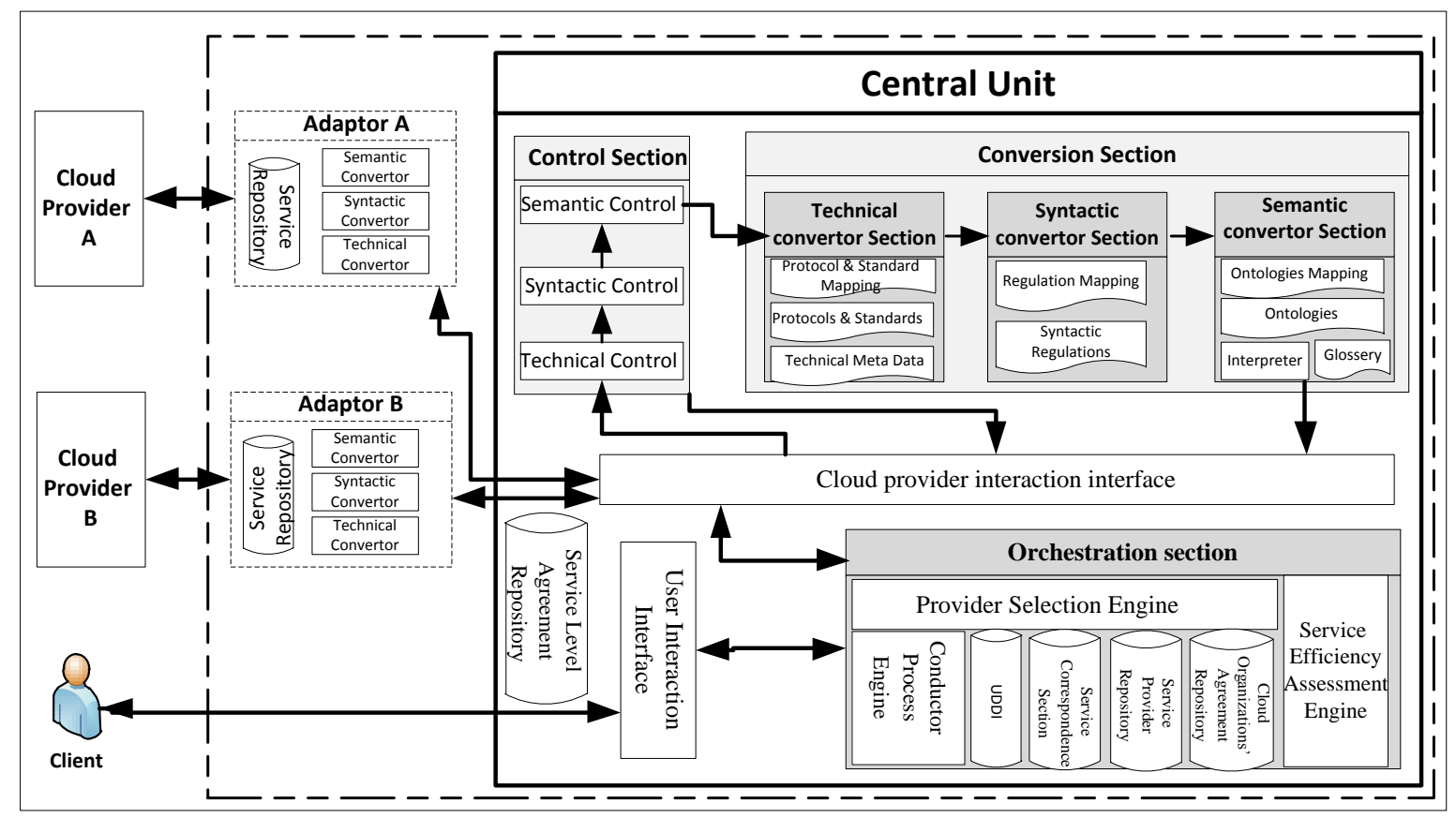

Fig. 3: Central Unit

3. The adaptor of the target cloud provider converts the received service to the required format by the target provider and delivers to it and thus the interoperability is established.

Fig. 2 outlines how to perform interoperability.

Another type of interaction is necessary in service composition. To create composite service, multiple services should be combined together and according to a composition schema which in orchestration layers of the central unit is defined by the conductor, they interact and cooperate with each other to achieve their objective. Interactive services might have been implemented in different areas and by different providers and may perform in different platforms. In the adaptor of each provider there is a part that is called service repository that the provider maintains WSDL of the services which can participate in orchestration along with their meta-data. There is also a public UDDI in the central unit, which contains the general information about the services of the providers which can participate in service composition. In the central unit, to orchestrate the services and to create composite services, there is a section which specifies the correspondence of the services and their orders. In this section languages like BPEL, which is an XML-based language, can be used in order to describe the logic of coordination and to control web services.

To create composite service first, the request of the user is analyzed and then the discovery and selection of the service according to the non-functional requirements like efficiency, quality and accuracy are done and in the end, the composite service is produced and the selection part of the provider selects the performer of the orchestration and then the composite service is executed and delivered to the client.

In establishing interoperability, as it is clear in the Fig.2, three levels are predicted. In the first level the adaptor of the source provider performs the required conversions on the service/group of services to be understandable for the central unit. The second level of conversions and mappings are performed in the central unit and in the third section

Conversions are performed in the target adaptor to make the service(s) ready for delivery to the target cloud provider.

Having at least two and at most three levels of conversions will let the central unit not to be responsible for all of the mappings and, in most cases, just play the role of a controller and on the other hand, without making any change in the structure and architecture of the cloud providers achieves Interoperability. This attitude towards conversions makes the scalability to be also supported.

\subsection{Components of the Central Unit}

The components of central unit are described as follows:

User interaction interface: links the central unit to the clients and their requests are sent through this interface to the cloud providers.

Cloud provider interaction interface: via this interface, the central unit will be linked to the cloud providers and service interaction will be done through this interface.

Service level agreement repository: All of the agreements that are made between the central unit and each provider and its adaptor for service exchange or delivery, will be maintained in this part.

Control section: the standards used by the source and target adaptor will be controlled here in order to specify whether conversions at this level is required or not.

Conversion section: this section includes technical, syntactic and semantic interfaces. Technical conversion interface deals with the issues related to the system connectivity and protocol conversions and through technical meta-data, protocols, standards and their mappings, provide the technical interoperability. Syntactic conversion interface deals with the issues related to the format of the services and their definition 
rules and using these defined rules for this section and the mapping among rules which are made by ontologies, makes the syntactic interoperability between cloud providers/organizations possible. Semantic conversion interface deals with the concept of the services and uses the defined translators and ontologies and the mapping among them to make the mutual understanding of the concepts of the services possible among the providers and thus, the semantic interoperability is created. In order to facilitate interoperability and make it more dynamic, sections of the glossary, syntactic rules, rules mapping and mapping of the ontologies are continuously updating new items and their mappings to the defined items.

Orchestration section: in this section, service compositions and also creating defined workflows by the clients using proposed basic services by the providers occurs. This section includes the following components:

- Conductor process engine: the sequence of performing and calling of the services in creating composite services are specified in this section. By using the languages like BPEL the sequence of services can be defined.

UDDI: is the central repository in which general information about the services of those providers which have the possibility to participate in creating composite services are maintained. Service description can be done by languages like WSDL. Meta-data of the services are also maintained in this section.

- Service correspondence section: in this section, correspondent services are mapped which facilitates the process of service composition and creating new workflows.

- Service provider Repository: in this section, the information of those providers for which the possibility of executing service orchestration is provided, are maintained.

- Cloud organizations' agreement Repository: in order to create a cloud organization and for the interactions among the participating providers, an agreement repository among the providers which are made by the central unit mediation is included here.

- Service efficiency assessment engine: when services are used by different providers or used in different compositions, in the end a survey by the engine from the section which used the services will be done about the quality of them based on the defined factors and the results will be analyzed and maintained in this section. In future, when the services are going to be used in orchestration, services with better quality and lower price will be selected. In fact, when special amount of service quality is desired, these values can be used and for this purpose different methods are proposed which are not in the framework of this research.

- Provider selection engine for orchestration: based on the information in the providers' repository, a provider which is capable of handling a workflow or executing a composite service is selected. Therefore, a bottleneck in the central unit or in a specific provider will be prevented.

\section{CASE STUDY}

Since the proposed solutions in the organizational architecture area are less provable through formal methods, their assessment and review are often carried out through case studies. For a better understanding of the efficiency of the proposed framework and a better statement of the problem and analysis, an understandable example is provided below. Nowadays, social networks like Facebook, Twitter, and Google Plus enjoy huge popularity, and many people throughout the world use these services. But the popularity of Facebook is more than the other social networks and its users are much more than the remaining networks. A lot of people are registered in all of these social networks. Normally, according to their priorities, people are more active in one of these networks and cannot be active in all of them, but many people like to have more interaction among their user accounts in their social networks and consequently for their activities to become visible to their friends in other social networks. In order to achieve this objective the proposed interoperability framework can be used and the responsibility of all of the interactions can be allocated to the central unit and the adaptors. At first, the client communicates with the central unit and requests the interaction among three services from different cloud providers, then according to the contract that is set between the client and the central unit, information is exchanged among the social networks that the client is registered in. for instance, the client states in the contract that all of the images, videos and statuses which he shares in Facebook be also visible to his friends in Twitter and Google Plus, therefore with being active in one social network he can be active in other networks too and provide his friends with his information in other social networks. As Fig. 4 shows, this possibility is provided through the adaptor and the central unit. Here, there will be at least two and at most three levels of conversion. At first, conversions are made by the adaptor for the central unit in order to make the information (e.g. images videos and statuses) understandable for it and then, according to the source and target provider which are selected by the client, controls are done in the central unit, and if necessary technical, syntactic and semantic conversions are done according to the standards of the target adaptor and are delivered to it and further conversions are done there in order to make the information understandable for the target social network. Using this method, the communication among several competing social networks will be provided and it makes the user able to interact with all of his friends in different social networks without spending much time. This possibility lets the users to be more active in the social network which is more user-friendly and at the same time keep their user accounts in other networks active. 


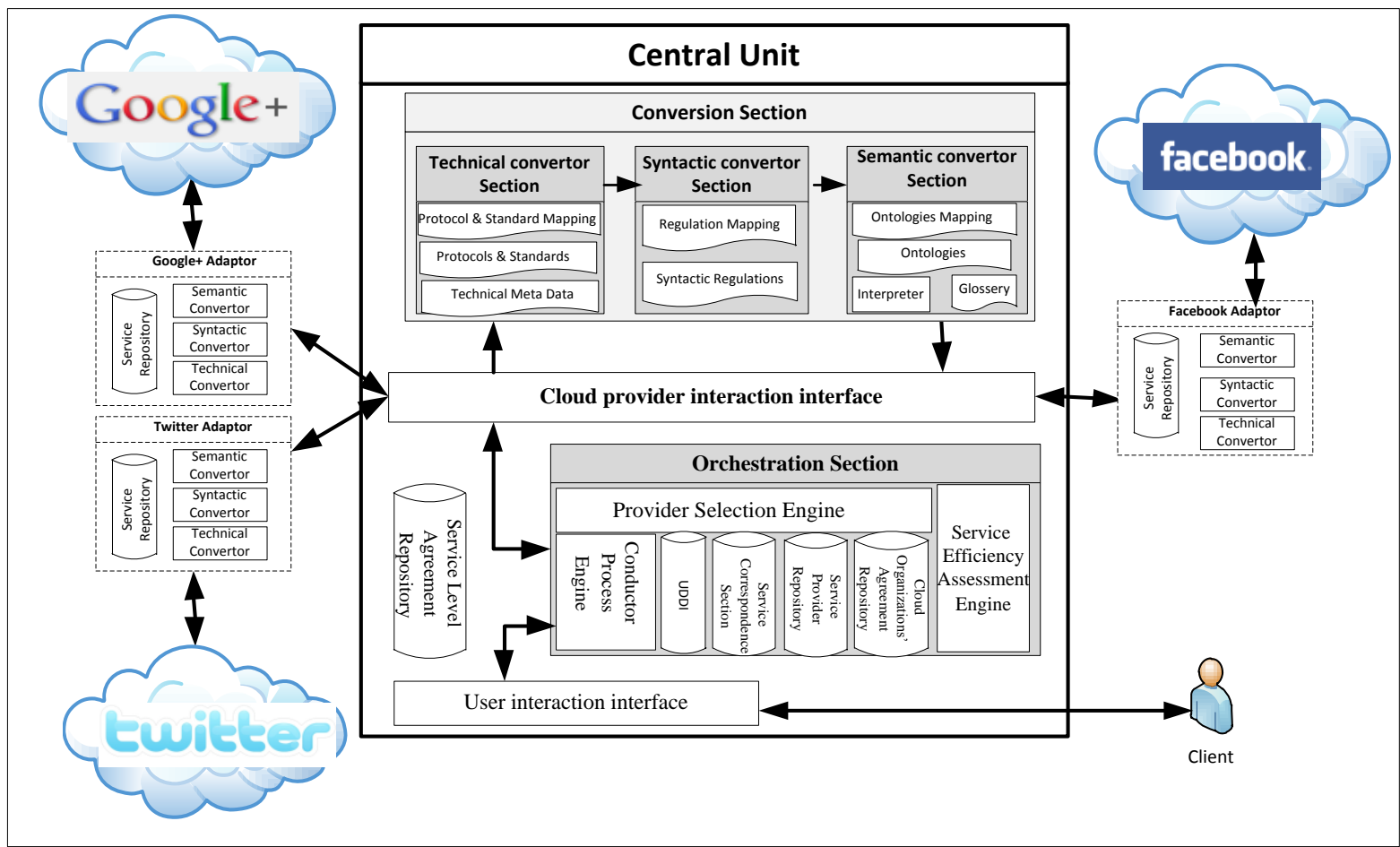

Fig. 4: Interaction among Social Networks

\section{CONCLUSION}

Considering the aforementioned descriptions, the specifications of the proposed framework is as follows:

- It will make the interaction and cooperation among providers possible without exerting any change in their basic structure

- It prevents client dependency on a specific provider and lets them migrate freely among the cloud providers and there will be no more specific client-provider dependency.

- This framework makes the scalability possible.

- It makes the definition of a new work process model and creation of an orchestration from the proposed services by different providers and achieving a new working objective, possible.

The proposed solution should be in a way that without making any or with little changes in providers make the interoperability possible and in fact it should be built upon the present platforms. The proposed framework provides this possibility with creating three levels of conversion and lets the services migrate and interact. The proposed interoperability solution has discussed the issue in the highest level and is a step towards solving the issue of Interoperability among Cloud providers and helps the architects continue in an organized way to do the implementation and establishing the desired architecture for the future works.
Based on authors' researches about interoperability among cloud providers, no specific works has been done in this area therefore we couldn't compare our research to other existing researches. For more description, researches done in the cloud interoperability area are mostly about defining standards and adhere.

\section{REFERENCES}

[1] Claybrook, B. 2011. Cloud Interoperability: Problems and best practice.

[2] Saikou, Y. 2010. Toward a Formal Theory of Interoperability, Old Dominion University.

[3] Chen, D. 2007 Framework of Enterprise Interoperability

[4] Parameswaran, A., and Chadha, A. 2009. Cloud Interoperability Standardization.

[5] Claybrook, B. 2009. Cloud Computing and Standardization, ETLabs Briefings VOV 7 NO.

[6] Distributed Management Task Force (DMTF): Open Cloud Standards Incubator, 2009. Available at: http://www.dmtf.org/ about/cloud-incubator.

[7] WANG, W., TOLK A., and WANG, WE., 2009. The Levels of Conceptual Interoperability Model

[8] Tambouris, F., Loutas, N., Peristeras, V., and Tarabanis K. 2008. The Role of Interoperability in eGovernance Applications.

[9] Chen, D., Vallespir, B., and Daclin, N., 2008. An Approach for Enterprise Interoperability Measurement, IMS-LAPS/GRAI, University of Bordeaux. 